\title{
Composição química e fitoquímica das farinhas da casca e da semente de lichias (Litchi chinensis Sonn) cultivar 'Bengal'
}

\author{
Chemical and phytochemical composition of flours from skin and seeds of 'Bengal' lychee \\ (Litchi chinensis Sonn)
}

\author{
Estela de Rezende Queiroz ${ }^{{ }^{*}}$ Celeste Maria Patto de Abreu \\ Cláudia Mendes dos Santos ${ }^{I}$ Anderson Assaid SimãoI
}

\section{RESUMO}

\begin{abstract}
Este estudo teve como objetivo determinar a composição química e fitoquímica das farinhas da casca e da semente de lichias (Litchi chinensis Sonn), família Sapindaceae, da cultivar 'Bengal'. A composição centesimal, o valor energético total, sólidos solúveis, $\mathrm{pH}$, acidez e os teores de compostos fenólicos, antocianinas, flavonoides, minerais, açúcares, vitamina C e a triagem fitoquímica foram determinados. A farinha da casca de lichia apresentou os maiores teores de flavonoides, vitamina $C$, compostos fenólicos, antocianinas, lipídios, proteinas, cinzas e fibras, enquanto a farinha da semente se destacou pelos teores de potássio, enxofre, cobre e zinco. Ambas as farinhas apresentaram resultado positivo para açúcares redutores, proteina e aminoácidos, taninos, catequinas, flavonoides, depsídeo e depsidonas. Somente a farinha da casca apresentou resultado positivo para alcaloides. As farinhas dos subprodutos da lichia apresentaram-se como fontes promissoras de nutriente e substâncias bioativas, no entanto suas propriedades biológicas devem ser estudadas.
\end{abstract}

Palavras-chave: aproveitamento de subprodutos, substâncias bioativas, minerais, triagem fitoquímica, Litchi chinensis Sonn.

\section{ABSTRACT}

This study had the objective to determine the chemical and phytochemical composition of flours from skin and seeds of 'Bengal' lychees (Litchi chinensis Sonn), Sapindaceae family. Proximate composition, total energy value, soluble solids, $\mathrm{pH}$, acidity and levels of phenolic compounds, anthocyanins, flavonoids, minerals, sugars, vitamin $C$ and the phytochemical screening were determined. Lychee skin flour presented the highest levels of flavonoids, vitamin $C$, phenolic compounds, anthocyanins, lipids, proteins, ash and fiber, while the seed flour stood out for the contents of potassium, sulfur, copper, and zinc. Both flours were positive for reducing sugars, protein and amino acids, tannins, catechins, flavonoids, depsides and depsidones. Only the skin flour showed a positive result for alkaloids. The flours made from lychee by-products were promising sources of nutrients and bioactive substances; however, their biological properties need to be further studied.

Key words: use of by-products, bioactive substances, minerals, phytochemical screening, Litchi chinensis Sonn.

\section{INTRODUÇÃO}

Além dos nutrientes essenciais, a maioria dos frutos possuem consideráveis quantidades de micronutrientes, tais como minerais, vitaminas e compostos fitoquímicos secundários. Nos últimos anos, maior atenção tem sido dada a estes compostos, uma vez que evidências apontam a importância desses micronutrientes para a saúde humana (ZHANG et al., 2013).

A lichia (Litchi chinensis Sonn) é um fruto tropical a subtropical, pertencente à família Sapindaceae, originário do sudeste da Ásia, que tem sido amplamente difundido como cultura econômica em diversos países, inclusive no Brasil. A produção brasileira da lichia ainda não está bem determinada e concentra-se, principalmente, na região Sudeste (SMARSI et al., 2011). Apesar da sua pouca expressão no mercado nacional, a lichia é um fruto não-climatérico, com alto valor comercial e muito apreciado pelos consumidores (XU et al., 2011; GUIMARÃES et al., 2013), o que contribui para a

IDepartamento de Química, Universidade Federal de Lavras (UFLA), Campus Universitário, CP 3037, 37200-000, Lavras, MG, Brasil. E-mail: estelaqueiroz@yahoo.com.br.*Autor para correspondência. 
sua grande aceitação no mercado, porém, ainda é pouco conhecida pelos brasileiros. A polpa da lichia é a parte comestível que pode ser consumida fresca ou processada enquanto que a casca e semente são descartadas, e, portanto, consideradas resíduos (XU et al., 2011, PRASAD et al., 2009).

Pesquisas relataram presença de proantocianidinas, flavonoides, esteroides, e sesquiterpenos na semente de lichia (XU et al., 2011), e que esta poderia ser utilizada como uma fonte de antioxidante natural, como ingrediente funcional ou conservante natural (PRASAD et al., 2009). Além disso, ela possui propriedades antihiperlipidêmica, antitumoral, antiplaquetária (XU et al., 2011). A casca contém grande quantidade de flavonoides e polifenóis (JIANG et al., 2013), com elevada atividade antioxidante, efeito cardioprotetor e atividades anticancerígenas (PRIOR \& GU, 2005), responsáveis pelos efeitos farmacológicos da casca de lichia (JIANG et al., 2013).

Produtos de origem vegetal apresentam reduzido tempo de armazenamento, devido ao alto teor de água. A secagem permite a redução de peso, o que diminui os custos de transporte, embalagem e armazenamento, além de estender o tempo de armazenamento. Adicionalmente, produtos farináceos são utilizados como ingredientes na indústria de alimentos, sobretudo na elaboração de pães e sopas. Assim, a produção de produto farináceo constitui-se numa alternativa para os subprodutos da lichia, favorecendo novos mercados. No entanto, as potenciais aplicações da farinha de casca e da semente de lichia dependem da sua composição. Desta forma, o objetivo deste estudo foi determinar a composição química e fitoquímica das farinhas da casca e da semente de lichias da cultivar 'Bengal'.

\section{MATERIAL E MÉTODOS}

Os frutos de lichia (Litchi chinensis Sonn), família Sapindaceae, da cv. 'Bengal' foram colhidos em pomar experimental situado no município de Lavras, Minas Gerais, safra agrícola 2012/2013 e selecionados de acordo com a uniformidade na coloração (casca vermelho-intenso), ausência de defeitos e tamanho médio.

Os frutos selecionados foram lavados, sanificados com dicloroisocianurato de sódio $200 \mu \mathrm{L}$ $\mathrm{L}^{-1}$ por $15 \mathrm{~min}$, pesados e separados em casca, polpa e semente. A casca e a semente foram secas em estufa a $45^{\circ} \mathrm{C}$, até peso constante, sendo necessários quatro dias para a secagem da casca e oito dias para a da semente. Estas foram moídas separadamente, obtendo-se a farinha da casca de lichia (FC) e a farinha da semente de lichia (FS), que foram armazenadas ao abrigo da luz, até a realização das análises.

A triagem de constituintes químicos foi realizada por reações químicas qualitativas simples, segundo metodologia descrita por MATOS (1997), utilizando os seguintes testes e reagentes: ácidos orgânicos (reativo de Pascová); açúcares redutores (reagentes de Fehling A e Fehling B); polissacarídeos (Lugol); proteínas e aminoácidos (reação de Molish e com ninidrina); taninos (reação com cloreto de ferro); catequinas (reação com metanol, vanilina 1\% e ácido clorídrico); flavonoides (reação com ácido clorídrico e fitas de magnésio); glicosídeos cardioativos (reativo de Kedde); sesquiterpenolactonas e outras lactonas (reação com cloridrato de hidroxilamina, hidróxido de sódio $10 \%$, em metanol, e ácido clorídrico); azulenos (reativo de Kaiser); carotenoides (reação com clorofórmio e ácido trifluoracético); esteroides e terpenoides (reação de Liebermann-Burchard); depsídeo e depsidonas (reação com éter etílico, metanol e cloreto de ferro 1\%); derivados da cumarina (teste da fluorescência, sob luz UV e hidróxido de sódio, seguida de extração em fase etérea); saponinas (índice de espuma após a agitação da solução neutralizada com carbonato de sódio); e, para os alcaloides, foram usados os reativos de Mayer (reação com iodo mercurato de potássio), de Dragendorff (reação com iodo bismutato de potássio) e de Bouchardat (reação com iodeto de potássio e iodo).

Os teores de lipídios (extrato etéreo), proteína bruta $(\mathrm{Nx} 6,25)$, cinzas e fibra alimentar foram quantificados utilizando o método descrito pela ASSOCIATION OF OFFICIAL AGRICULTURAL CHEMISTRY - AOAC (2012). O carboidrato foi determinado por diferença entre 100 e a soma das demais frações da composição centesimal, a umidade calculada por gravimetria e o valor energético total (VET) pela soma das calorias fornecidas por carboidratos, lipídios e proteínas, multiplicando-se seus valores em gramas pelos fatores de Atwater: $4 \mathrm{kcal}, 9 \mathrm{kcal}$ e $4 \mathrm{kcal}$, respectivamente.

Os sólidos solúveis totais foram determinados por refratometria digital e expressos em ${ }^{\circ}$ Brix, conforme AOAC (2012). O pH foi determinado por potenciometria em eletrodo de vidro, segundo AOAC (2012), e a acidez titulável foi determinada por titulação com solução de $\mathrm{NaOH} 0,1 \mathrm{~N}$, usando fenolftaleína como indicador.

Os açúcares foram extraídos, em etanol $95 \%$, evaporados a $80^{\circ} \mathrm{C}$ e submetidos à hidrólise ácida com $\mathrm{HCl}$ concentrado, em ebulição, por 15 minutos, conforme LANE \& EYNON (1934). 
Estes foram quantificados pelo método Somogyi (NELSON, 1944), através da redução do íon cúprico a cuproso, que reage com o reativo arsênio-molibídico, formando óxido de molibdênio, cuja concentração é proporcional à quantidade de açúcares na amostra.

Os extratos de vitamina $\mathrm{C}$ foram preparados, sob agitação por $15 \mathrm{~min}$, em banho ultrassônico, em ácido oxálico $0,5 \%$, acrescido de 0,1g de Kieselguhr e quantificado por espectrometria, segundo STROHECKER \& HENNING (1967).

Os compostos fenólicos foram extraídos em metanol 50\%, em condensador de refluxo, por três vezes consecutivas, a $80^{\circ} \mathrm{C}$. Os extratos foram reunidos e doseados usando o reagente de FolinDenis, a 760nm (AOAC, 2012). Os resultados foram expressos em mg de ácido gálico $100 \mathrm{~g}^{-1}$ de amostra.

Os flavonoides foram extraídos com metanol $80 \%$, em condensador de refluxo, a $80^{\circ} \mathrm{C}$ e quantificado por espectrometria UV-VIS a $510 \mathrm{~nm}$ (ZHISHEN et al., 1999), usando rutina como padrão.

As antocianinas totais foram determinadas pelo método de FULEKI \& FRANCIS (1968). Estas foram extraídas em metanol $95 \%$, acidificado com ácido clorídrico $1,5 \mathrm{M}$, na proporção de $85: 15 \mathrm{v} / \mathrm{v}$, por 24 horas, sob refrigeração, quantificadas por espectrofotometria de UV-Vis e expressas em $\mathrm{g}$ de cianidina 3-glicosídeo $100 \mathrm{~g}^{-1}$ de amostra.

Os teores de minerais (fósforo, potássio, cálcio, magnésio, enxofre, cobre, manganês, zinco e ferro) nas farinhas foram doseados segundo MALAVOLTA et al. (1997), após digestão nitroperclórica.

Foi utilizado o delineamento inteiramente casualizado, DIC, sendo dois tratamentos farinha da casca e farinha da semente de lichia, analisados em quatro repetições por tratamento. Os resultados foram expressos em média \pm desvio padrão.

\section{RESULTADOS E DISCUSSÃO}

A lichia (Litchi chinensis Sonn), família Sapindaceae, apresentou peso médio de $16,36 \pm 0,25 \mathrm{~g}$, dos quais $29,27 \pm 0,28 \%$ eram cascas e $20,09 \pm 0,52 \%$ sementes. As umidades encontradas para a FC e FS

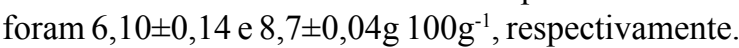

Com a realização da triagem fitoquímica, constatou-se a presença ou ausência de compostos provenientes do metabolismo secundário do vegetal, nas farinhas da casca e semente de lichia, através da formação de precipitados e pelo surgimento de coloração e espuma. Não se detectaram polissacarídeos, glicosídeos cardíacos, sesquiterpenlactona e outras lactonas, carotenoides, esteroides e triterpenoides, derivados de cumarina e alcaloides (Mayer) nas farinhas analisadas. A presença de açúcares redutores, proteína e aminoácidos, taninos, catequinas, flavonoides, depsídeo e depsidonas foi observada em ambas as farinhas. Azuleno, ácidos orgânicos e alcaloide (Dragendorff e Bouchardat) foram detectados na FC. Somente na FS observou-se a formação de espuma estável, revelando a presença de saponina espumídica.

No que tange à aplicação terapêutica, os taninos são conhecidos por sua atividade antioxidante in vivo e ex vivo e atividade hipocolesterolêmica (TIAN et al., 2012), protegendo os tecidos da peroxidação lipídica. Inúmeras atividades biológicas estão associadas aos flavonoides, como funções antioxidante, anti-inflamatória e anticancerígena (KSOURI et al., 2013), enquanto que os depsídeos e depsidonas apresentam propriedades antioxidantes (CHOMCHEON al., 2009), analgésicas e antipiréticas (OKUYAMA et al., 1995). Aos alcaloides, são atribuídas propriedades farmacológicas variadas, sendo usados como analgésicos, antioxidantes, vasorrelaxantes e antitumorais, o que causou um aumento no interesse por potenciais fontes desses compostos (CUI et. al., 2006). Às saponinas espumídicas, têm sido associadas atividades hemolítica, antiviral, anti-inflamatória (KSOURI et al., 2013).

A composição centesimal e o valor energético total (VET) das farinhas da lichia estão apresentados na tabela 1 . Os teores de umidade da FC e da FS, respectivamente, foram de $6,10 \pm 0,14$ e $8,7 \pm 0,04 \mathrm{~g} 100 \mathrm{~g}^{-1}$. Baixos teores de umidade são importantes para conservação das farinhas, pois impedem o desenvolvimento microbiano e as reações químicas e enzimáticas que promovem alterações indesejáveis. O limite máximo de umidade estabelecido para a farinha de trigo, pela legislação brasileira, é de 15\% (BRASIL, 2005). As umidades da FC e FS encontravam-se abaixo desse limite.

Os teores de proteínas e de lipídios foram baixos, sobretudo na FS. A FC apresentou, também, os maiores teores de cinzas e fibras $(7,11 \pm 1,09$, $10,08 \pm 0,21, \quad 3,25 \pm 0,52$ e $19,88 \pm 0,66 \mathrm{~g} \quad 100 \mathrm{~g}^{-1}$, respectivamente), enquanto que na FS o teor de carboidratos e o VET foram superiores ao da FC. Estes dados demonstram que esta farinha pode ser considerada uma boa fonte energética. A composição centesimal e o VET relativos às farinhas da casca e da semente de lichia foram semelhantes aos reportados por QUEIROZ et al. (2012), que quantificaram na casca da lichia teores de lipídios, proteínas, cinzas, fibras e carboidratos de 6,$97 ; 10,86 ; 2,17 ; 18,89$ e 
Tabela 1 - Composição centesimal, em g $100 \mathrm{~g}^{-1}$, da farinha da casca (FC) e da farinha da semente (FS) de lichias.

\begin{tabular}{lll}
\hline Parâmetros & \multicolumn{1}{c}{ FC } & FS \\
\hline Lipídios & $7,11 \pm 1,09$ & $4,73 \pm 0,27$ \\
Proteínas & $10,08 \pm 0,21$ & $5,33 \pm 0,27$ \\
Cinzas & $3,25 \pm 0,52$ & $1,75 \pm 0,05$ \\
Fibras & $19,88 \pm 0,66$ & $4,75 \pm 0,27$ \\
Carboidratos & $59,67 \pm 0,43$ & $83,44 \pm 0,43$ \\
VET $^{*}$ & $343,04 \pm 4,94$ & $397,66 \pm 2,83$ \\
\hline
\end{tabular}

* Valor energético total expresso em kcal $100 \mathrm{~g}^{-1}$ de farinha.

$61,11 \mathrm{~g} 100 \mathrm{~g}^{-1}$, respectivamente, e na semente de 2,77 ; 4,$83 ; 1,44 ; 4,33$ e $86,63 \mathrm{~g} 100 \mathrm{~g}^{-1}$, respectivamente.

$\mathrm{Na}$ tabela 2, encontram-se os teores dos compostos físico-químicos e químicos analisados na FC e na FS. Observa-se que o teor de sólidos solúveis totais da FC foi superior ao da FS $(20,0 \pm 2,31$ e $15,0 \pm 1,21^{\circ} \mathrm{Brix}$, respectivamente). Teores de sólidos solúveis totais elevados são comuns em farinhas de frutos, pois a retirada da umidade concentra os açúcares e ácidos orgânicos. O teor de sólidos solúveis totais da farinha de acerola $\left(47,5^{\circ} \mathrm{Brix}\right)$, encontrado por Aquino et al. (2010), foi superior ao da FC e da FS. Constata-se, também, que a acidez titulável da FC foi superior à da FS e, consequentemente, seu $\mathrm{pH}$ foi menor. Esses resultados se assemelham aos reportados por QUEIROZ et al. (2012) para a casca (acidez $0,59 \%$ e $\mathrm{pH} 4,70$ ) e para a semente de lichia (acidez 0,23\%; pH 6,3).

Tabela 2 - Composição físico-química e química da farinha da casca (FC) e da farinha da semente (FS) de lichias.

\begin{tabular}{|c|c|c|}
\hline & $\mathrm{FC}$ & FS \\
\hline $\begin{array}{l}\text { Sólidos Solúveis } \\
\left({ }^{\circ} \text { Brix }\right)\end{array}$ & $20,00 \pm 2,31$ & $15,00 \pm 1,21$ \\
\hline $\begin{array}{l}\text { Acidez titulável } \\
\text { (\% ácido málico) }\end{array}$ & $0,64 \pm 0,04$ & $0,23 \pm 0,06$ \\
\hline $\mathrm{pH}$ & $4,38 \pm 0,04$ & $6,31 \pm 0,06$ \\
\hline $\begin{array}{l}\text { Açúcares totais } \\
\left(\mathrm{g} 100 \mathrm{~g}^{-1}\right)\end{array}$ & $10,40 \pm 0,45$ & $15,13 \pm 0,10$ \\
\hline $\begin{array}{l}\text { Vitamina C } \\
\left(\mathrm{mg} 100 \mathrm{~g}^{-1}\right)\end{array}$ & $295,69 \pm 8,62$ & $57,54 \pm 1,39$ \\
\hline 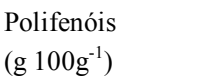 & $6,36 \pm 0,63$ & $3,89 \pm 0,28$ \\
\hline $\begin{array}{l}\text { Antocianinas } \\
\left(\mathrm{mg} 100 \mathrm{~g}^{-1}\right)\end{array}$ & $99,55 \pm 2,25$ & $6,21 \pm 0,11$ \\
\hline $\begin{array}{l}\text { Flavonoides } \\
\left(\mathrm{mg} 100 \mathrm{~g}^{-1}\right)\end{array}$ & $82,16 \pm 9,56$ & $46,74 \pm 7,70$ \\
\hline
\end{tabular}

Nota-se também que a FC apresenta acidez titulável superior à encontrada na FS $(0,64 \pm 0,04 \%$ e $0,23 \pm 0,06 \%$, respectivamente), enquanto a FS apresenta o $\mathrm{pH}$ mais elevado $(6,31 \pm 0,06)$. Estes resultados se assemelham aos reportados por QUEIROZ et al. (2012), que obtiveram, na casca, acidez e pH de $0,59 \%$ e 4,70 , respectivamente, e, na semente, teores de $0,23 \%$ e 6,3 , respectivamente

A FS apresentou o maior teor de açúcar total $\left(15,13 \pm 0,10 \mathrm{~g} \quad 100 \mathrm{~g}^{-1}\right)$, enquanto a $\mathrm{FC}$ se destacou pelos teores de antocianinas, compostos fenólicos, vitamina $\mathrm{C}$ e flavonoides $(99,55 \pm 2,25 \mathrm{mg}$ $100 \mathrm{~g}^{-1}, 6,36 \pm 0,63 \mathrm{~g} 100 \mathrm{~g}^{-1}, 295,69 \pm 8,62 \mathrm{mg} 100 \mathrm{~g}^{-1} \mathrm{e}$

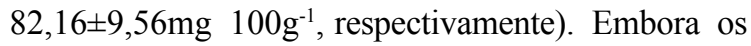
conteúdos de vitamina $\mathrm{C}$, antocianinas, compostos fenólicos e flavonoides encontrados na FC tenham sido superiores aos obtidos na semente, pode-se considerar que a FS também concentra expressivos teores destes fitoquímicos, reconhecidos por sua atividade antioxidante. Assim, as farinhas dos subprodutos da lichia, sobretudo a da casca, mostraram-se como fontes destes fitoquímicos, podendo ser utilizadas para o enriquecimento de alimentos. Os conteúdos de flavonoides totais na FC e na FS foram superiores aos reportados em subprodutos de manga, maracujá, goiaba e caju $\left(26,47 ; 43,08 ; 31,41\right.$ e 44,91mg $100 \mathrm{~g}^{-1}$ de matéria seca, respectivamente) (SILVA et al. 2014).

Os teores de minerais das duas farinhas estão apresentados na tabela 3 . AFS se destacou pelos teores de $\operatorname{KeS}\left(0,59 \pm 0,02\right.$ e $0,37 \pm 0,01 \mathrm{~g} 100 \mathrm{~g}^{-1}$, respectivamente), além de $\mathrm{Cu}$ e $\mathrm{Zn}\left(17,70 \pm 4,28\right.$ e $13,15 \pm 2,41 \mathrm{mg} \mathrm{kg}^{-1}$, respectivamente), enquanto que na $\mathrm{FC}$ os outros macros e microminerais encontravam-se em maior proporção.

Os valores de IDR (Ingestão Diária Recomendada) para o potássio, magnésio, ferro, zinco, manganês, cobre e cálcio são 4,6g, 260mg, $14 \mathrm{mg}, 7 \mathrm{mg}, 2,3 \mathrm{mg}, 900 \mu \mathrm{g}$ e $1,3 \mathrm{~g}$, respectivamente, para indivíduos adultos (FREIRE et al., 2012). Dessa forma, a ingestão de 100 gramas de FC e de FS cobrirá, respectivamente, 12 e $13 \%$; 92 e $27 \%$; 24 e $17 \% ; 16$ e $19 \%$; 52 e $41 \% ; 150$ e $197 \% ; 17$ e $11 \%$ das IDRs desses minerais.

Os resultados observados neste estudo sugerem que as farinhas da casca e da semente de lichia, apesar de apresentarem composições químicas distintas, são ingredientes potenciais para a utilização em rações comerciais e no preparo de pães, bolos e biscoitos ou ainda incorporadas a alimentos, contribuindo, dessa forma, com a IDR dos minerais e com as necessidades energéticas e nutricionais. Assim, o aproveitamento das farinhas dos subprodutos da lichia poderá ser realizado individualmente, 
Tabela 3 - Teores de macrominerais ( $\mathrm{P}, \mathrm{K} \mathrm{Ca}, \mathrm{Mg}$ e S, expressos

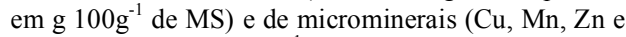
$\mathrm{Fe}$, expressos em $\mathrm{mg} \mathrm{kg}^{-1}$ ) na farinha da casca (FC) e na farinha de semente (FS) de lichias.

\begin{tabular}{lll}
\hline & FC & \multicolumn{1}{c}{ FS } \\
\hline $\mathrm{P}$ & $0,17 \pm 0,01$ & $0,13 \pm 0,01$ \\
$\mathrm{~K}$ & $0,54 \pm 0,02$ & $0,60 \pm 0,02$ \\
$\mathrm{Ca}$ & $0,22 \pm 0,02$ & $0,14 \pm 0,01$ \\
$\mathrm{Mg}$ & $0,25 \pm 0,01$ & $0,07 \pm 0,01$ \\
$\mathrm{~S}$ & $0,30 \pm 0,03$ & $0,37 \pm 0,01$ \\
$\mathrm{Cu}$ & $13,50 \pm 0,93$ & $17,70 \pm 2,28$ \\
$\mathrm{Mn}$ & $12,75 \pm 0,33$ & $9,62 \pm 0,98$ \\
$\mathrm{Zn}$ & $11,58 \pm 0,18$ & $13,15 \pm 0,41$ \\
$\mathrm{Fe}$ & $34,03 \pm 1,55$ & $23,77 \pm 2,36$ \\
\hline
\end{tabular}

contudo a utilização destas farinhas misturadas pode ser relevante. Entretanto, vale ressaltar que novos estudos devem ser conduzidos, buscando avaliar os efeitos da utilização destas farinhas em rações e como ingredientes alimentares.

\section{CONCLUSÃO}

As farinhas da casca e semente de lichia demonstram possuir composições químicas e nutricionais que possibilitam suas utilizações na dieta humana e na indústria de alimentos, podendo contribuir como fontes alternativas de nutrientes e substâncias bioativas.

A farinha da casca de lichia apresenta elevados teores de fibras alimentares e proteínas, enquanto que a farinha da semente se destaca pelos teores de carboidratos. Ambas as farinhas são fontes do mineral cobre e mostram a presença dos fitoquímicos flavonoides, vitamina $\mathrm{C}$, compostos fenólicos e antocianinas. No entanto, para que estas farinhas sejam aproveitadas, é necessária a realização de novos estudos sobre suas propriedades biológicas.

\section{AGRADECIMENTOS}

Os autores agradecem ao Conselho Nacional de Desenvolvimento Científico e Tecnológico (CNPq), Coordenação de Aperfeiçoamento de Pessoal de Nível Superior (CAPES) e Fundação de Amparo à Pesquisa do estado de Minas Gerais (FAPEMIG).

\section{REFERÊNCIAS}

AOAC (ASSOCIATION OF OFFICIAL ANALYTICAL CHEMISTRY). Official methods of analysis. 19.ed. Gaithersburg, 2012. 3000p.

AQUINO, A.C.M.S. et al. Avaliação físico-química e aceitação sensorial de biscoitos tipo cookies elaborados com farinha de resíduos de acerola. Revista do Instituto Adolfo Lutz, v.69, p.379-386, 2010.

BRASIL. Ministério de Agricultura, Pecuária e Abastecimento. Instrução normativa $\mathrm{n}^{\circ} \mathrm{8}, 02$ jun. 2005. Regulamento Técnico de Identidade e Qualidade da Farinha de Trigo. Diário Oficial da República Federativa do Brasil, Brasília, DF, 03 jun. 2005, Seção 1, n.105, p.91.

CHOMCHEON, P. et al. Aromatase inhibitory, radical scavenging and antioxidant activities of depsidones and diaryl ethers from the endophytic fungus Corynespora cassiicola L36. Phytochemistry, v.70, p.407-413, 2009. Disponível em: <http://dx.doi.org/10.1016/j. phytochem.2009.01.007>. Acesso em: 04 set. 2013. doi: 10.1016/j. phytochem.2009.01.007.

CUI, W. et al. Potencial cancer chemopreventive activity of simple isoqinolines, 1 benziylisoquinolines, and protoberberines. Phytochemistry, v.67, p.70-79, 2006. Disponível em: <http:// dx.doi.org/10.1016/j.phytochem.2005.10.007>. Acesso em: 25 maio 2013. doi: $10.1016 /$ j.phytochem.2005.10.007.

FREIRE, J.M. et al. Avaliação de compostos funcionais e atividade antioxidante em farinhas de polpa de goiabas. Revista Brasileira de Fruticultura, v.34, p.847-852, 2012. Disponível em: <http:// dx.doi.org/10.1590/S0100-29452012000300026>. Acesso em: 25 mar. 2013. doi: 10.1590/S0100-29452012000300026.

FULEKI, T.; FRANCIS, F.J. Extraction and determination of total anthocyanins in cranberries. Journal of Food Science, v.33, p.72-77, 1968. Disponível em: <http://onlinelibrary.wiley.com/ doi/10.1111/j.1365-2621.1968.tb00887.x/abstract>. Acesso em: 25 ago. 2014. doi: 10.1111/j.1365-2621.1968.tb00887.x.

GUIMARÃES, J.E.R. et al. Ácido cítrico e quitosana na conservação de lichias 'bengal'. Revista Brasileira de Fruticultura, v.35, p.730-737, 2013. Disponível em: <http:// dx.doi.org/10.1590/S0100-29452013000300009>. Acesso em: 22 out. 2013. doi: 10.1590/S0100-29452013000300009.

LANE, J.H.; EYNON, L. Determination of reducing sugars by Fehling solution with methylene blue indicator. London : Norman Rodger, 1934. 8p.

JIANG, G. et al. Identification of a novel phenolic compound in litchi (Litchi chinensis Sonn.) pericarp and bioactivity evaluation. Food Chemistry, v.136, p.563-568, 2013. Disponível em: <http:// dx.doi.org/10.1016/j.foodchem.2012.08.089>. Acesso em: 02 nov. 2013. doi: $10.1016 /$ j.foodchem.2012.08.089.

KSOURI, W.M. et al. LC-ESI-TOF-MS identification of bioactive secondary metabolites involvedin the antioxidant, antiinflammatory and anticancer activities of the edible halophyte Zygophyllum album Desf. Food Chemistry, v.139, p.10731080, 2013. Disponível em: <http://dx.doi.org/10.1016/j. foodchem.2013.01.047>. Acesso em: 25 ago. 2014. doi: 10.1016/j. foodchem.2013.01.047.

MALAVOLTA, E. et al. Avaliação do estado nutricional de plantas: princípios e aplicações. Piracicaba : Potafos, 1997. 308p.

MATOS, F.J.A. Introdução a fitoquímica experimental. 2.ed. Fortaleza : UFC, 1997. 141p. 
NELSON, N.A. A photometric adaptation of the Somogyi method for the determination of glucose. Journal of Biology Chemistry, v.153, p.375-380, 1944. Disponível em: <http://www.jbc.org/ content/153/2/375.full.pdf>. Acesso em: 25 ago. 2014.

OKUYAMA, E. et al. Usnic acid and diffractic acid as analgesic and antipyretic components of Usnea diffracta. Planta Medica, v.61, p.113-115, 1995.

PRASAD, K.N. et al. Identification of phenolic compounds and appraisal of antioxidant and antityrosinase activities from litchi (Litchi sinensis Sonn.) seeds. Food Chemistry, v.116, p.1-7, 2009. Disponível em: <http://dx.doi.org/10.1016/j. foodchem.2009.01.079>. Acesso em: 20 jun. 2013. doi: 10.1016/j. foodchem.2009.01.079.

PRIOR, R.L.; GU, L. Occurrence and biological significance of proanthocyanidins in the American diet. Phytochemistry, v.66, p.2264-2280, 2005. Disponível em: <http://www.sciencedirect. com/science/article/pii/S0031942205001408\#>. Acesso em: 25 ago. 2014. doi:10.1016/j.phytochem.2005.03.025.

SILVA, L.M.R. et al. Quantification of bioactive compounds in pulps and by-products of tropical fruits from Brazil. Food Chemistry, v.143, p.398-404, 2014. Disponível em: <http:// dx.doi.org/10.1016/j.foodchem.2013.08.001>. Acesso em: 25 ago. 2014. doi: 10.1016/j.foodchem.2013.08.001

STROHECKER, R.; HENNING, H.M. Analisis de vitaminas: metodos comprobados. Madrid : Paz Montalvo, 1967. 428p.

TIAN, Y. et al. High molecular weight persimmon tannin is a potent antioxidant both ex vivo and in vivo. Food Research International, v.45, p.26-30, 2012. Disponível em: <http://www.
sciencedirect.com/science/article/pii/S0963996911005667>. Acesso em: 25 ago. doi:10.1016/j.foodres.2011.10.005.

QUEIROZ, E.R. et al. Constituintes químicos das frações de lichia in natura e submetidas à secagem: potencial nutricional dos subprodutos. Revista Brasileira de Fruticultura, v.34, p.11741179, 2012. Disponível em: <http://dx.doi.org/10.1590/S010029452012000400026>. Acesso em: 25 ago. 2014. doi: 10.1590/ S0100-29452012000400026.

SMARSI, R.C. et al. Efeito da adubação nitrogenada na produção de mudas de lichieira. Revista Ceres, v.58,n.1,p.129-131,2011. Disponível em: <http://dx.doi.org/10.1590/S0034-737X2011000100020>. Acesso em: 25 ago. doi: 10.1590/S0034-737X2011000100020.

$\mathrm{XU}, \mathrm{X}$. et al. A novel cyclopropyl-containing fatty acid glucoside from the seeds of Litchi chinensis. Fitoterapia, v.82, p.485488, 2011. Disponível em: <http://dx.doi.org/10.1590/S0034737X2011000100020>. Acesso em: 25 ago. doi: 10.1016/j. fitote.2011.01.001.

ZHANG, R. et al. Phenolic profiles and antioxidant activity of litchi pulp of different cultivars cultivated in Southern China. Food Chemistry, v.136, p.1169-1176, 2013. Disponível em: <http:// dx.doi.org/10.1016/j.foodchem.2012.09.085>. Acesso em: 25 ago. doi: 10.1016/j.foodchem.2012.09.085.

ZHISHEN, J. et al. The determination of flavonoid contents in mulberry and their scavenging effects on superoxide radicals. Food Chemistry, v.64, p.555-559, 1999. Disponível em: <http://www. sciencedirect.com/science/article/pii/S0308814698001022\#>. Acesso em: 25 ago. doi: 10.1016/S0308-8146(98)00102-2. 